\title{
Off-center explosions of Chandrasekhar-mass white dwarfs: an explanation of super-bright type la supernovae?
}

\author{
W. Hillebrandt ${ }^{1}$, S. A. Sim ${ }^{1}$, and F. K. Röpke ${ }^{1,2}$ \\ 1 Max-Planck-Institut für Astrophysik, Karl-Schwarzschild-Str. 1, 85741 Garching, Germany \\ e-mail: [wfh;ssim; fritz] @mpa-garching.mpg.de \\ 2 Department of Astronomy and Astrophysics, University of California, Santa Cruz, 1156 High Street, Santa Cruz CA 95064, USA
}

Received 15 January 2007 / Accepted 13 February 2007

ABSTRACT

\begin{abstract}
Context. The recent discovery of a very bright type Ia supernova, SNLS-03D3bb (三SN 2003fg), in the Supernova Legacy Survey (SNLS) has raised the question of whether super-Chandrasekhar-mass white-dwarf stars are needed to explain such bright explosions. Progenitors of this sort could form by mergers of pairs of rather massive white dwarfs. Binary systems of two white dwarfs in close orbit, where their total mass significantly exceeds the Chandrasekhar mass, have not yet been found. Therefore SNLS-03D3bb could establish the first clear case of a double-degenerate progenitor of a (peculiar) type Ia supernovae. Moreover, if this interpretation is correct, it casts some doubt on the universality of the calibration relations used to make SNe Ia distance indicators for cosmology. Aims. We aim to evaluate the case for a super-Chandrasekhar-mass progenitor for SNLS-03D3bb in light of previous theoretical work on super-Chandrasekhar-mass explosions. Furthermore, we propose an alternative scenario involving only a Chandrasekhar-mass progenitor.

Methods. We present a theoretically motivated critical discussion of the expected observational fingerprints of super-Chandrasekharmass explosions. As an alternative, we describe a simple class of aspherical Chandrasekhar-mass models in which the products of nuclear burning are displaced from the center. We then perform simple radiative transfer calculations to predict synthetic lightcurves for one such off-center explosion model.

Results. In important respects, the expected observational consequences of super-Chandrasekhar-mass explosions are not consistent with the observations of SNLS-03D3bb. We demonstrate that the lopsided explosion of a Chandrasekhar-mass white dwarf could provide a better explanation.
\end{abstract}

Key words. stars: supernovae: general - stars: white dwarfs - hydrodynamics - methods: numerical

\section{Introduction}

Type Ia supernovae (SNe Ia), i.e. stellar explosions whose spectra show no signatures of hydrogen but do show lines from elements of intermediate-mass nuclei and the iron group, have received considerable attention recently, mainly because of their use as distance indicators and as tools for determining cosmological parameters.

Although the luminosity of nearby, well-observed SNe Ia varies by more than a factor of ten, empirical correlations between their absolute magnitude and distance-independent properties, such as the shape of their light curves, provide the means to calibrate their distances. Several groups have used this approach to estimate the luminosity of SNe Ia at redshifts up to about 1.5 (Riess et al. 1998; Perlmutter et al. 1999; Tonry et al. 2003; Riess et al. 2004; Astier et al. 2006). Although they used different calibration procedures and different distant SN samples, they all agree that $\mathrm{SNe}$ Ia at high redshift appear fainter than they should in a Universe that is matter-dominated today, suggesting that the Universe started to accelerate its expansion when it was about half its present age. This behavior can be interpreted by using cosmologies that include a positive cosmological constant or a "dark energy" with negative pressure.

The empirical calibrations can be justified because they reduce the scatter in SN Ia Hubble diagrams, but an implicit assumption in all of them is that all SNe Ia have similar progenitors, namely almost spherically-symmetric white dwarfs at or near the Chandrasekhar mass, consisting of carbon and oxygen and disrupted by a thermonuclear combustion wave. In this scenario, the (bolometric) peak luminosity is proportional to the amount of newly synthesized radioactive ${ }^{56} \mathrm{Ni}$ ("Arnett's rule", Arnett 1982) and the observed peak-luminosity/lightcurve shape relation is the result of secondary effects, such as different opacities and/or thermal structures. It would be difficult to conceive ways of obtaining the observed simple relationships if the progenitors were to span a wide range of masses, as could be the case for objects formed by the merger of two white dwarfs. Therefore, the presently favored evolutionary track to explosion is accretion onto the white dwarf from a main-sequence or subgiant companion star rather than from a merger.

An obvious prediction of the Chandrasekhar-mass model is a limiting peak bolometric magnitude for SNe Ia of about $M_{\mathrm{bol}}=$ -20 , corresponding to $1.4 M_{\odot}$ of ${ }^{56} \mathrm{Ni}$, the Chandrasekhar mass. This is confirmed by a recent parameter study (Woosley et al. 2006) that shows that only extreme Chandrasekhar-mass models can reach $B$-band magnitudes close to -20 , i.e., models in which it is assumed that almost the entire star is burned. In reality, of course, the Ni mass has to be lower, allowing for at least about $0.3 M_{\odot}$ in intermediate-mass nuclei, as seen in the spectra of $\mathrm{SNe}$ Ia around maximum light, and taking into consideration that 
nuclear burning will also produce stable iron-group nuclei. In fact, typical deflagration Chandrasekhar-mass explosion models produce about $0.7 M_{\odot}$ or less of radioactive $\mathrm{Ni}$, and even for a detonation incinerating the entire white dwarf to iron-group nuclei, some of the ash would be in the form of stable iron and nickel.

Therefore it came as a big surprise when, as part of the SNLS on April 24, 2003, an SN Ia was discovered at a redshift of $z=0.244$ with a peak luminosity in the rest frame $V$ band of $20.5 \mathrm{mag}$, corresponding to an absolute magnitude of $M_{V}=-19.94 \pm 0.06$ for a standard cosmology (Howell et al. 2006). Using "Arnett's" rule (Arnett 1982), Howell et al. infer a mass of radioactive $\mathrm{Ni}$ of $(1.29 \pm 0.07) M_{\odot}$ and conclude that SNLS-03D3bb was most likely the result of the explosion of a rapidly spinning super-Chandrasekhar-mass white dwarf. A similar conclusion was reached by Jeffery et al. (2006). In fact, in their model the mass of the progenitor has to be about $2 M_{\odot}$ in order to account for the photospheric velocity of SNLS-03D3bb - about $8000 \mathrm{~km} \mathrm{~s}^{-1}$ on day +2 after $B$-maximum (Howell et al. 2006; see the discussion in the supplementary material to their paper). Howell et al. (2006) also briefly mention the possibility of an asymmetric explosion but conclude that this effect would be too small to account for the high luminosity of SNLS03D $3 \mathrm{bb} / \mathrm{SN} 2003 \mathrm{fg}$.

In this Letter we first discuss this scenario in the light of explosion models of super-Chandrasekhar-mass white dwarfs and argue that the photometric and spectroscopic data seem to exclude this possibility for SNLS-03D3bb. We then present an alternative scenario in which nuclear burning in a Chandrasekhar-mass white dwarf ignites off-center and drives a lopsided explosions. Here, the high apparent luminosity of SNLS-03D3bb would result from a nickel blob rising inside the star towards the observer. Synthetic lightcurves for a toy model are presented to support our interpretation. In a forthcoming paper, the consequences for supernova cosmology will be discussed.

\section{Super-Chandrasekhar-mass SN la models and their predictions}

In principle, a close binary system of two massive white dwarfs can merge and form a new rapidly spinning, degenerate star that considerably exceeds the Chandrasekhar limit in which the final configuration is stabilized by rotation. It was shown by Müller \& Eriguchi (1985) that such stars can have masses well in excess of $2 M_{\odot}$ and angular momenta sufficiently low to avoid the various rotational instabilities, provided that they rotate differentially. Additionally, it is not excluded that accretion onto a normal white dwarf might have similar results under certain circumstances (Yoon \& Langer 2005).

If such a star loses angular momentum or the angular momentum is redistributed closer to rigid rotation, it will become unstable, contract, and ignite its nuclear fuel. Central ignition and off-center ignition are conceivable, as well as a spontaneous detonation or a deflagration. Steinmetz et al. (1992) carried out an extended parameter study of detonations of differentially and rigidly rotating super-Chandrasekhar-mass $\mathrm{C}+\mathrm{O}$ white dwarfs in 2D assuming axi-symmetry. This is a fair approximation since it was shown by Benz et al. (1990) by means of SPH simulations that after a few revolutions the merging white-dwarf pair approaches an almost axially-symmetric hydrostatic configuration. The hope of Steinmetz et al. (1992) was that, due to the lower densities of the rapidly spinning models, a detonation would find enough white dwarf material at sufficiently low densities, $\rho \lesssim 10^{7} \mathrm{~g} \mathrm{~cm}^{-3}$, that carbon and oxygen would burn to silicon and not to nickel.

To that end, the results of the study were disappointing. The models did produce amounts of Ni that could easily bring them into agreement with very bright supernovae such as SN 1991T (or SNLS-03D3bb). However, no matter whether central or offcenter explosions were considered, or rigidly or differentially rotating progenitors of different masses, the amount of Si produced never exceeded a few $10^{-2} M_{\odot}$. This is too little, by a considerable margin, to explain the strong Si II line seen in SN Ia spectra, including SNLS-03D3bb. The reason is simply that the detonation does not leave enough time for the star to expand, and the amount of low-density progenitor material is small, even in rapidly spinning white dwarfs. Moreover, the expansion velocities for all cases considered were much higher than those inferred for SNLS-03D3bb from the spectrum observed at day +2 . Consequently, a prompt detonation of a super-Chandrasekharmass white dwarf is clearly ruled out.

More recently, Pfannes (2006) computed models of both deflagrations and detonations of super-Chandrasekhar-mass white dwarfs. The code he used is essentially that of Reinecke et al. (2002), modified and improved by Röpke (2005) and Schmidt et al. (2006) for pure deflagrations and by Maier \& Niemeyer (2006) to handle detonation in $\mathrm{C}+\mathrm{O}$ fuel. As far as detonations are concerned, Pfannes's work confirms the earlier results of Steinmetz et al. (1992). Although, in a few cases and for certain rotation laws, he finds that up to $15 \%$ of the mass is burned to intermediate-mass nuclei, barely enough to produce the $\mathrm{Si}$ II feature seen in the spectrum of SNLS-03D3bb, the velocity (10000 to $20000 \mathrm{~km} \mathrm{~s}^{-1}$ ) of this Si would be far too high.

Deflagration models of super-Chandrasekhar-mass white dwarfs, on the other hand, fall short of producing the amount of $\mathrm{Ni}$ required for bright $\mathrm{SNe}$ Ia. Typical differentially rotating super-Chandrasekhar-mass models predict even less Ni than non-rotating or rigidly rotating Chandrasekhar-mass models. The reason is easy to understand. Differential rotation suppresses the growth of the Rayleigh-Taylor instability that is the prerequisite for turbulence and that, in turn, determines the rate of fuel consumption. Differential rotation (needed to increase the mass beyond the Chandrasekhar limit) thus decreases the burning rate. Finally, the spectra expected from these models would fit those of neither normal nor of bright SNe Ia (Pfannes 2006).

The only other possible super-Chandrasekhar-mass case, which has not been investigated until now, is that of a deflagration-to-detonation transition in a rapidly spinning white dwarf. However, it is not clear whether one would get much more $\mathrm{Ni}$ in this case than from a pure deflagration. The start of such a model would be the deflagration phase discussed previously. The deflagration-to-detonation transition could occur in unburned $\mathrm{C}+\mathrm{O}$ near the center at densities where complete nuclear burning would take place. But it could also start at low density, thus resulting in incomplete burning that would produce intermediate-mass nuclei and not the iron group. Which of these two possibilities may occur, if either, will have to be determined from future simulations.

\section{Lopsided Chandrasekhar-mass explosions}

Here we suggest an alternative model to explain the luminosity of very bright $\mathrm{SNe}$ Ia. The model we propose has two ingredients: first, it needs about $1 M_{\odot}$ of radioactive $\mathrm{Ni}$, and second, the radioactive Ni responsible for the optical display of the supernova has to be distributed unevenly on large scales. 


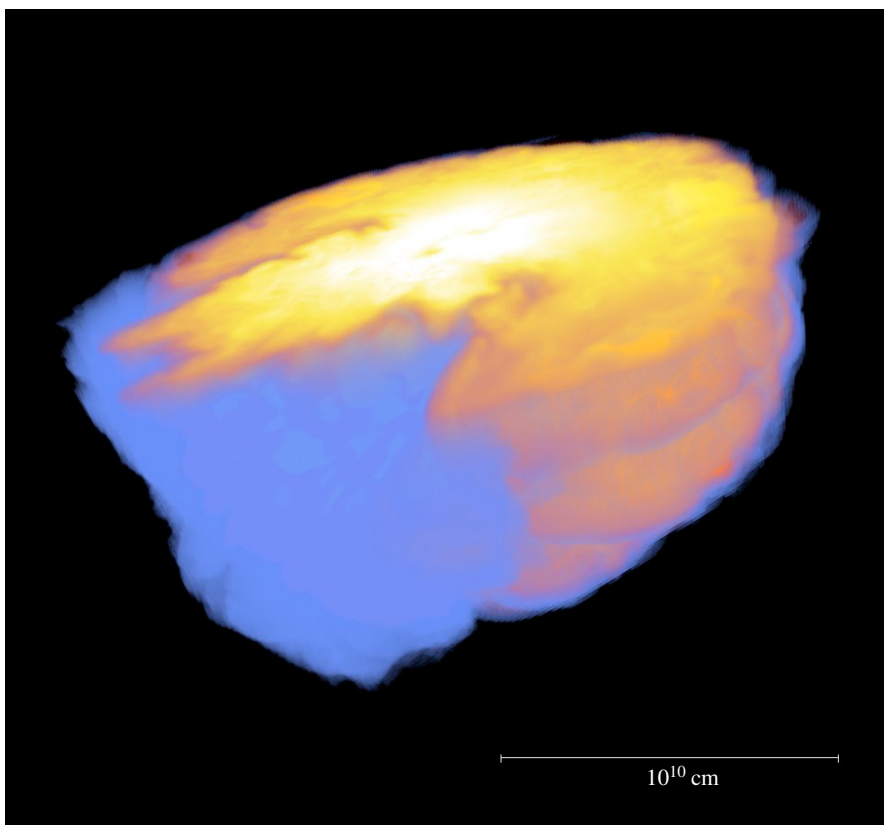

Fig. 1. Chemical structure of a pure-deflagration off-center 3D explosion of a Chandrasekhar-mass $\mathrm{C}+\mathrm{O}$ white dwarf $10 \mathrm{~s}$ after ignition. Half of the star is shown. Volume rendered is the density multiplied by the respective mass fractions: iron-group elements (mostly ${ }^{56} \mathrm{Ni}$, shown in yellow/brown); unburned $\mathrm{C}+\mathrm{O}$ (shown in blue).

Numerical simulations have shown ways in which this could happen in a real supernova. The rather high mass of Ni could result from a deflagration-to-detonation transition (DDT) taking place when the star has expanded to densities around $10^{7} \mathrm{~g} \mathrm{~cm}^{-3}$ during an initial deflagration phase (Gamezo et al. 2005; Golombek \& Niemeyer 2005; Röpke \& Niemeyer 2007). The asymmetry of the Ni distribution could be an intrinsic property of a DDT at one or a few points (Livne 1999), or might be a consequence of an already lop-sided deflagration (Calder et al. 2004; Röpke et al. 2006). (See Fig. 1, model 3T2d200 of Röpke et al. 2006 as an example of a significantly aspherical deflagration model. Note, however, that while this deflagration was sufficient to unbind the star, no DDT was assumed for this particular case.) In principle, asymmetric explosions might also result if, after an off-center deflagration phase, the white dwarf stays gravitationally bound and a surface wave triggers a secondary detonation (Plewa et al. 2004; Plewa 2006, but see Röpke et al. 2006). However, since 'surface-detonation models' like those of Plewa (2006) predict expansion velocities in excess of those inferred from the observed spectrum of SNLS-03D3bb (Howell et al. 2006), that particular scenario does not seem appropriate here.

In order to investigate the observable consequences of a lopsided distribution of radioactive nickel in a rather general way, we used a class of simply-parameterized models in which a spherical blob of nickel-rich material is placed in a homologously expanding spherical supernova. Our models explore two free parameters: the mass of $\mathrm{Ni}$ and its displacement. Guided by the numerical simulations discussed earlier, we assume that the center-of-mass of the nickel blob is displaced from the origin along the $+z$-direction by an arbitrary velocity shift $(\Delta v)$. Within the nickel-rich blob, a fixed initial mass fraction of ${ }^{56} \mathrm{Ni}$ is adopted $(f)$; it is assumed that there is no ${ }^{56} \mathrm{Ni}$ outside the blob. Although simplistic, these models provide a convenient and readily understandable tool for quantifying effects.

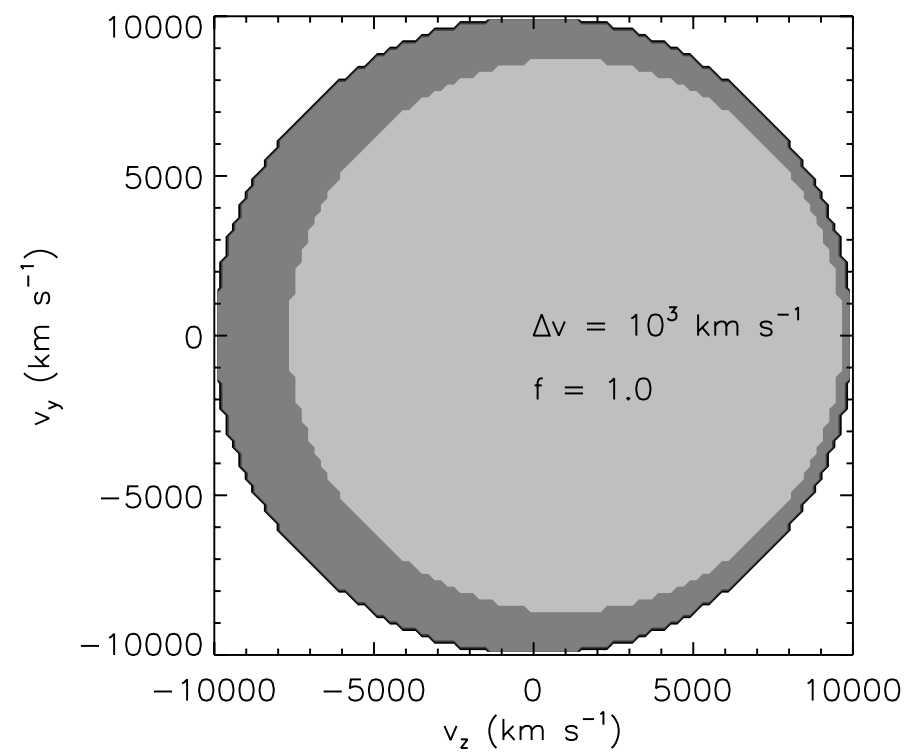

Fig. 2. Slice through the $y-z$ plane showing the distribution of ${ }^{56} \mathrm{Ni}$ adopted in the model. The dark grey area indicates the regions in which there is no ${ }^{56} \mathrm{Ni}$, while the light grey area, the center of which is displaced by $\Delta v=10^{3} \mathrm{~km} \mathrm{~s}^{-1}$ from the origin, contains pure ${ }^{56} \mathrm{Ni}(f=1)$. The model is symmetric under rotation about the $z$-axis.

In the context of SNLS-03D3bb, we discuss one particular realization of the model. A slice through this particular geometry (in velocity space) is shown in Figure 2 . The model has a total mass of $1.4 M_{\odot}$, an initial ${ }^{56} \mathrm{Ni}$-mass of $0.89 M_{\odot}$, a maximum expansion velocity of $10^{4} \mathrm{~km} \mathrm{~s}^{-1}$, and uniform mass density. The nickel blob parameters are $f=1$ and $\Delta v=10^{3} \mathrm{~km} \mathrm{~s}^{-1}$. Wider discussion of these lopsided models and their implications will be presented by Sim et al. (in preparation).

Using the Monte Carlo radiative transfer code described by Sim (2007), bolometric lightcurves were computed for various viewing angles to the model. A uniform, grey-absorption cross-section of $0.1 \mathrm{~cm}^{2} \mathrm{~g}^{-1}$ was adopted; this simple treatment is expected to maximize the apparent viewing-angle dependence of the light curve and so allows a limit on the scale of any such effect to be established. In Figure 3, two of the computed light curves are shown: first, the angle-averaged light curve (this is the lightcurve obtained by averaging over those seen from a large number of randomly oriented lines-of-sight) and secondly, the light curve recorded by a distant observer on the $+z$-axis.

As one would expect, the angle-averaged light curve from the model cannot account for the observed peak brightness of SNLS-03D3bb; the peak magnitude is only -19.58 mag compared to $M_{\mathrm{bol}}=-19.87 \pm 0.06 \mathrm{mag}$ as reported by Howell et al. (2006). However, the lopsided nature of the model causes the light curve to be dependent on the viewing angle such that, if viewed along the direction in which the nickel blob is displaced, the peak is nearly 0.3 mag brighter. This effect brings us to consistency with the observed peak brightness of SNLS-03D3bb.

A valid question, of course, is whether our model meets the velocity constraint of SNLS-03D3bb. Of course, we cannot answer this question for a "real" 3D off-center DDT of the sort we propose because hydrodynamical simulations for such a model have not yet been performed. Most likely the velocity will be similar to what is obtained in published (less asymmetric) DDT models. For example, model (a) of Gamezo et al. (2005) predicts a maximum expansion velocity of the outermost layers of about $10000 \mathrm{~km} \mathrm{~s}^{-1}$ for a total Ni mass of $0.8 M_{\odot}$. On day +2 (when 


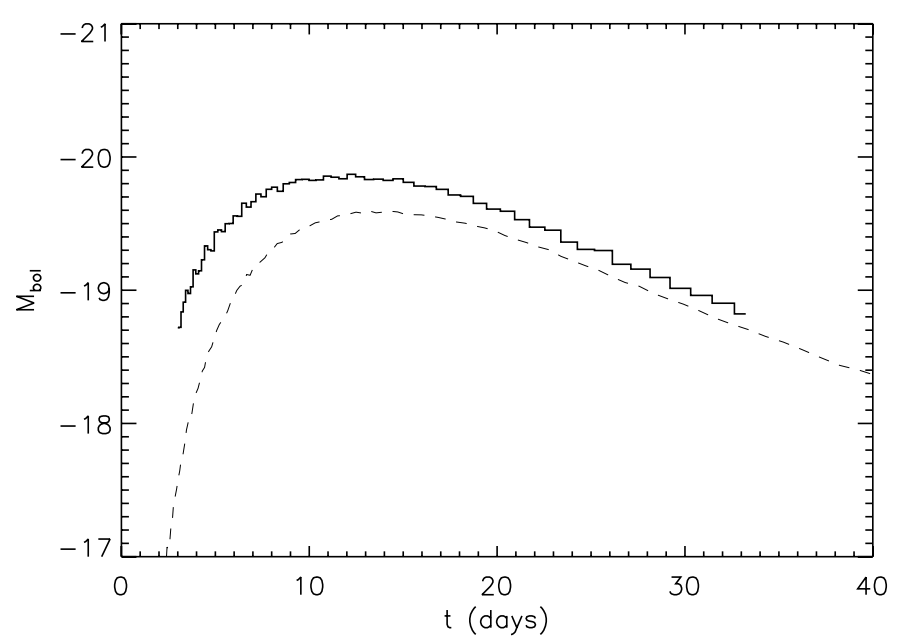

Fig. 3. Bolometric light curves computed from the model. The dashed line is the angle-averaged light curve and the solid histogram indicates the light curve as viewed from the $+z$-direction.

the spectrum of SNLS-03D3bb was taken) and later, most SNe Ia show constant $\mathrm{Si}$ velocity, indicating that the $\mathrm{Si}$-layer is outside the photosphere. For the Gamezo et al. model, this means that the Si velocity at that epoch would be $\sim 8000 \mathrm{~km} \mathrm{~s}^{-1}$, in fair agreement with SNLS-03D3bb.

We can be a little more precise for the toy model we have used here. We assumed a maximum velocity of $10000 \mathrm{~km} \mathrm{~s}^{-1}$. (This is somewhat arbitrary, however, and in principle we could have adopted a lower or a higher velocity.) As in the DDT model discussed above, this peak ejection velocity does not cause a problem because the majority of the ejecta, even in the narrow region above the Ni-bubble on the front hemisphere of the model, has lower velocities than the maximum value. Also, projection effects will always cause the line-of-sight velocity deduced from the spectrum to be lower than the true velocity. In fact, most of the material in front of the Ni-bubble has a typical line-of-sight velocity of only $6000 \mathrm{~km} \mathrm{~s}^{-1}$. Thus, although we cannot make detailed predictions for the spectrum on day +2 without undertaking considerably more sophisticated radiative transfer calculations, we conclude that the velocities reported by Howell et al. (2006) do not exclude our model.

The model considered here is tuned in a number of respects. First, the combination of $\mathrm{Ni}$ mass $\left(0.89 M_{\odot}\right)$ and geometry (Fig. 2) is arranged to produce close to the maximum effect possible. Increasing the mass of the Ni blob further does not allow a peak magnitude brighter than $\sim 20$ to be obtained from any direction; although more $\mathrm{Ni}$ makes the angle-averaged lightcurve brighter, it does this at the expense of suppressing the angular variation since the mass of the surrounding shell becomes lower. For lower Ni masses, it is possible to obtain more significant angular variations, but the absolute magnitude rapidly becomes too faint.

Second, the treatment of opacity is also extreme. The adopted cross-section $\left(0.1 \mathrm{~cm}^{2} \mathrm{~g}^{-1}\right)$ may be optimistically high for material in which significant nuclear burning has not taken place. Since it is the opacity in the outer regions that leads to the angular variations in the lightcurve, it is likely that even these outer regions must be burned to at least intermediate mass elements. Conversely, the adopted cross-section may be too low for the region inside the Ni-rich blob. If the opacity in this region were higher, the lightcurve would rise more slowly and have a correspondingly dimmer peak. To investigate the effects of the opacity treatment in detail would require much more complex non-grey, non-LTE radiation transport, which lies beyond the scope of this letter, but which is vital for fully understanding these effects in the future.

\section{Conclusions}

In light of these considerations, we draw the following conclusions. It may be possible to observe lightcurves with a peak brightness close to that of SNLS-03D3bb/SN 2003fg from supernovae with a lopsided distribution of radioactive material, despite the total mass of this material being only $\geq 0.9 M_{\odot}$; thus this model is a viable alternative to the proposed superChandrasekhar theory.

However, since the conditions on a simple off-center explosion model that can explain the brightness of SNLS-03D3bb are quite tight, we can make several predictions from this hypothesis, which may be testable via future observations. First, these objects must be rare since both a moderately-high nickel mass $\left(\geq 0.9 M_{\odot}\right)$ and rather special viewing direction are required. Second, SNLS-03D3bb lies very close to the limit of brightness that can be readily achieved - thus any supernovae more than one or two tenths of a magnitude brighter can not be explained in this way. Third, the asymmetries postulated in our model should leave markers in the nebular spectra, such as a net redshift of the lines of intermediate-mass elements. And finally, our calculations suggest that extremely bright $\mathrm{SNe}$ Ia should have shorterthan-average light-curve rise times.

\section{References}

Astier, P., Guy, J., Regnault, N., et al. 2006, A\&A, 447, 31

Arnett, W. D. 1982, ApJ, 253, 785

Benz, W., Cameron, A. G. W., Press W. H., \& Bowers, R. L. 1990, ApJ, 348, 647 Calder, A. C., Plewa, T., Vladimirova, N., Lamb, D. Q., \& Truran, J. W. 2004, [arXiv:astro-ph/0405162]

Gamezo, V. N., Khokhlov, A. M., \& Oran, E. S. 2005, ApJ, 623, 337

Golombek, I., \& Niemeyer, J. C. 2005, A\&A, 438, 611

Howell, D.A., Sullivan, M., Nugent, P., et al. 2006, Nature, 443, 309

Jeffery, D. J., Branch, D., \& Baron, E. 2006, [arXiv: astro-ph/0609804]

Livne, E. 1999, ApJ, 527, L97

Maier, A., \& Niemeyer, J.C. 2006, A\&A, 451, 207

Müller, E., \& Eriguchi, Y. 1985, A\&A, 152, 325

Perlmutter, S., Aldering, G., Goldhaber, G., et al. 1999, ApJ, 517, 565

Pfannes, J. M. M. 2006, Ph. D. Thesis, Universität Würzburg

Plewa, T., Calder, A. C., \& Lamb, D. Q. 2004, ApJ, 612, L37

Plewa, T. 2006, ApJ, in press, [arXiv: astro-ph/0611776]

Reinecke, M., Hillebrandt, W., \& Niemeyer, J. C. 2002, A\&A, 386, 936

Riess, A. G., Filippenko, A. V., Challis, P., et al. 1998, AJ, 116, 1009

Riess, A. G., Strolger, L.-G., Tonry, J., et al. 2004, ApJ, 607, 665

Röpke, F. K. 2005, A\&A, 432, 969

Röpke, F. K., \& Niemeyer, J. C. 2007, A\&A, 464, 683

Röpke, F. K., Woosley, S. E., \& Hillebrandt, W. 2006, ApJ, submitted, [arXiv: astro-ph/0609088]

Schmidt, W., Niemeyer, J.C., Hillebrandt, W., \& Röpke, F. K. 2006, A\&A, 450, 283

Sim, S. A. 2007, MNRAS, 375, 154

Steinmetz, M., Müller, E., \& Hillebrandt, W. 1992, A\&A, 254, 177

Tonry, J. L., Schmidt, B. P., Barris B., et al. 2003, ApJ, 594, 1

Woosley, S. E., Kasen, D., Blinnikov, S., \& Sorokina, E. 2006, ApJ, submitted, [arXiv:astro-ph/0609562]

Yoon, S.-C., \& Langer, N. 2005, A\&A, submitted, 435, 967 